\title{
Interferometric coronography for the DARWIN space mission - Laboratory demonstration experiment
}

\author{
M. Ollivier ${ }^{1}$, J.-M. Mariotti ${ }^{\dagger 2}$, A. Léger ${ }^{3}$, P. Sékulic ${ }^{3}$, J. Brunaud ${ }^{3}$, and G. Michel ${ }^{2}$ \\ 1 Max-Planck-Institut für Astronomie, Königstuhl 17, 69245 Heidelberg, Germany \\ 2 DESPA, Observatoire de Paris Meudon, France \\ 3 Institut d'Astrophysique Spatiale, Orsay, France
}

Received 7 September 2000 / Accepted 7 March 2001

\begin{abstract}
The DARWIN mission aims at directly detecting exoplanets, particularly telluric ones, around nearby stars and at performing a spectral analysis of their potential atmosphere. The major difficulty in achieving this goal is the huge luminosity contrast between the star and its possible planet(s). The mission concept is based on the star light rejection by nulling interferometry. This concept has never been experimentally demonstrated with high values of rejection $\left(10^{5}\right)$ in the thermal infrared where DARWIN will observe. In this paper, we describe a laboratory test-bed that uses a $\mathrm{CO}_{2}$ laser at $10.6 \mu \mathrm{m}$ to achieve this goal in a monochromatic case. We describe its principle, its sub-systems, their realizations and the first results we obtained with this interferometer.
\end{abstract}

Key words. instrumentation: interferometers - methods: laboratory - techniques: interferometric - infrared: general

\section{Introduction}

The search for extra-solar planets is one of the most exciting fields in present-day astrophysics. We are living at an epoch where progress in instrumentation is beginning to allow the detection of other planets around nearby stars.

The first step was reached when Wolszczan and Frail announced the discovery of a planetary system around a pulsar (PSR 1257+12) (Wolsczczan 1992) but a real advance occurred when Mayor and Queloz announced the discovery of a planet around the solar-type star 51Peg (Mayor \& Queloz 1995). At present, more than 50 other planets $^{1}$ have been discovered (Schneider 2000), mainly using a radial velocity technique. Even though this method has shown its efficiency (determination of the period, semimajor axis, lower limit of the planet mass, multiplicity of the system), it is an indirect method of detection, and cannot give spectral information about the planet itself.

Direct methods of detection such as imaging or spectroscopy should in the near future make it possible to get information about the planets' composition. Several space missions are presently under study to reach that goal: DARWIN in Europe (Léger et al. 1993; Léger et al. 1996; Fridlund et al. 2000), and TPF in the United States (Woolf et al. 1998). The instrumental concept for such

Send offprint requests to: M. Ollivier, e-mail: ollivier@mpia-hd.mpg.de

1 New planet discoveries may have been announced since the manuscript was sent to the editors. missions should allow high angular resolution as well as high dynamic range observations. For example the observation of an Earth-like planet orbiting around a solar type star located at $10 \mathrm{pc}$ from the sun subtends an angle of 0.1 arcsec as well as dynamic range $\Delta m$ around 17 in the $N$ band.

One possible instrument that fulfills these requirements is the Bracewell interferometric coronograph (Bracewell 1978), or instruments based on similar concepts (Angel et al. 1986; Léger et al. 1993). The specificity of all these instruments is to cancel the flux in the direction they are pointed in (stellar direction) while the flux from an off-axis direction is transmitted. Thus, they are well suited for the detection of faint star companions.

In order to be efficient, the on-axis extinction should be comparable to the contrast between the star and its companion (about $710^{6}$ at $10 \mu \mathrm{m}$ ). The extinction is quantified by the rejection rate, written $\rho$ and defined as the ratio of the constructive to the destructive interference flux. An ideal interferometer has thus an infinite rejection rate for a point source at infinity, because the on-axis extinction is total. Due to optical ripples and coating inhomogeneities, the extinction of a real interferometer is never perfect and thus it has a limited rejection rate (the dark fringe is never completely dark).

Several experiments to demonstrate the validity of this concept are under way (Serabyn 1999; Hinz et al. 2000; Morgan et al. 2000) but at present, no experimental demonstration of high rejection nulling interferometry 
$\left(\rho>10^{4}\right)$ has been proposed in the thermal infrared. We already have quantified the requirements for a $10^{6}$ rejection rate nulling interferometer (Ollivier \& Mariotti 1997; Ollivier 1999). In this paper, we describe an experimental setup that should reach that level of extinction in a monochromatic case at $\lambda \sim 10 \mu \mathrm{m}$. In the first section, we summarize the requirements needed to reach such a high rejection rate. In the second section, we describe the experimental interferometer we have built, the principles behind it and the fabrication and performance of its individual components. In the last section, we present the first results obtained with it.

\section{High rejection nulling interferometry: Theoretical requirements}

Let us consider a two wavefront pupil interferometer, set in flat field (the interference state is uniform over the whole area of interference). Theoretically, two wavefronts give perfect constructive (respectively destructive) interference if, on each point of the beam-combiner, they have the same amplitude, phase (respectively same phase but one wavefront is $\pi$-shifted) and polarization state. This requires a perfect matching of the two wavefronts. Every differential distortion in amplitude, in phase or in the polarization state leads to a local modification of the interference state. If the interferometer is set in a dark flat field, it leads to a light leak through the interference pattern. In this paper, we will consider only the amplitude and phase distortions of the wavefronts because we are working in a monochromatic case with a linearly polarized laser.

One can easily show that a $10^{6}$ rejection rate could only be reached with a two wavefront interferometer if (Ollivier 1999):

i) the mean phase difference, including optical path difference (OPD), does not exceed $210^{-3}$ radian;

ii) the mean differential intensity difference does not exceed $0.4 \%\left(\Delta I / I<410^{-3}\right)$.

These conditions, in terms of optical constraints, lead to the following optical requirements, estimated by numerical simulation (Ollivier \& Mariotti 1997):

- less than $0.0002 \%$ of the optical surface covered by dust (0 transmission points);

- less than $0.5 \%$ inhomogeneity in the coating reflection coefficient;

- Phase control better than $\lambda / 3000$, from OPD to surface roughness scales ( $\lambda$ is the working wavelength);

- Pointing control of each telescope within 1/100th of the full width of the Airy disk.
Even if they are individually achievable, satisfying all of these requirements simultaneously would lead to a costly system.

Numerical simulations and calculations have already shown that these requirements could strongly be reduced if an optical filtering of the Airy figure of each telescope of the interferometer is performed, because:

i) each telescope individually does not resolve the system star-planet, so all the information is contained inside the Airy disk;

ii) a major part of phase and amplitude defects are high spatial frequency defects with effects outside the Airy disk. Thus, they are eliminated well by optical filtering.

Optical filtering can be performed by a pinhole (Ollivier \& Mariotti 1997) or by single-mode waveguides (Mennesson et al. 2001). In the first case, a simple spatial filtering has to be considered: the higher the spatial frequency of the defect, the better the filtering efficiency. In the second case the filtering effect is obtained by the fact that only one mode propagates through a singlemode waveguide. The other modes of the corrupted wavefront (including tilt, higher order aberrations and substrate roughness) are eliminated through the propagation. The amplitude of the wavefront at the waveguide output depends on the coupling efficiency of the corrupted wavefront to the propagating mode, while the phase is a function of the mean phase before injection into the waveguide.

The first case will later be called "spatial filtering" and the second "modal filtering".

\section{Experimental setup: Strategy and practical realization}

We have built an experimental setup to validate these simulations and to test the efficiency of optical filtering with different devices, pinholes and dielectric waveguides. The measurement of the rejection rate allows us to quantify the filtering efficiency. The strategy adopted for the setup is simple:

\footnotetext{
- we control low spatial frequency defects that are not well corrected by pinhole filtering (mainly, OPD and tip-tilt for phase and global intensity of each beam for amplitude);

- we optically filter high frequency amplitude and phase defects.
}

The demonstration experiment uses an amplitude division Mach-Zehnder type interferometer in which several elements have been modified (Fig. 1).

We took into account neither the chromaticity of the rejection rate, nor its feasibility over a large range of wavelengths (typically $6-18 \mu \mathrm{m}$ for the DARWIN space mission). The wavelength of $10.6 \mu \mathrm{m}$ has been chosen because 


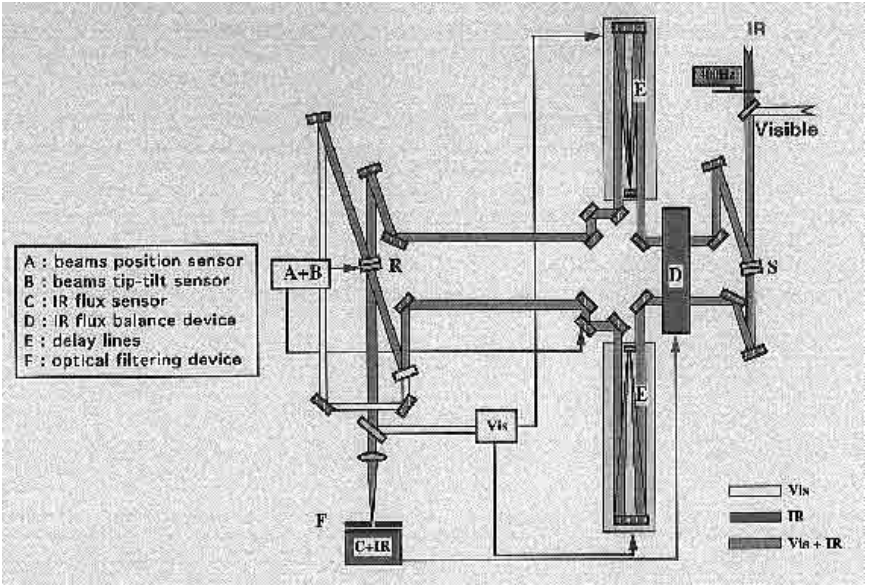

Fig. 1. Demonstration experiment scheme. On a classical Mach-Zehnder structure, several devices have been put: a beam intensity equalizing device, delay lines, a tip-tilt correction device and an optical filtering device located after the recombination and before the detection

it was located roughly in the middle of DARWIN's spectral range. In addition, single mode lasers exist at this wavelength and can provide a higher signal than the thermal background ${ }^{2}$. A red visible laser beam $(\lambda \sim 630 \mathrm{~nm})$ is superimposed on the infrared laser beam. We thus have a star model with visible and infrared monochromatic radiation. The ratio of 17 between visible (red) and infrared wavelengths allows a huge increase in the accuracy of the settings. For instance, a path difference of $\lambda_{\mathrm{IR}} / 3000$ corresponds approximatively to a path difference of $\lambda_{\mathrm{Vis}} / 200$.

All the optical elements used in this experiment should allow the propagation of visible and infrared wavelengths. With that goal, the refractive optical elements (beamsplitters and windows) are in zinc selenide (ZnSe) while the mirrors, generally in Zerodur, are coated with a single layer of gold deposited on a chrome coated blank to increase the quality of the gold layer.

Let us now consider, the different parts of the setup.

\subsection{Light sources}

The infrared source is a $\mathrm{CO}_{2}$ laser, lasing on the P20 line, pumped by radio-frequency, linearly polarized (single polarization), transverse and longitudinal single-mode, with a maximum power of $250 \mathrm{~mW}$ at $10.59 \mu \mathrm{m}$. The frequency stability of the laser is better than $10 \mathrm{kHz}$ (for $T<10 \mu \mathrm{s}$ ). The source is tunable in a range of $\pm 100 \mathrm{MHz}$. It is placed at one edge of the optical table, and its beam is expanded by a 3 lens optical device. The final pupil is circular, with a radius of $12 \mathrm{~mm}$ and cuts the Gaussian laser beam at

\footnotetext{
2 The $300 \mathrm{~K}$ background is the major source of noise at $10 \mu \mathrm{m}$ on the Earth (for the demonstration experiment) or in its neighbourhood. This problem is less critical for DARWIN because the observatory will be located in space where a $40 \mathrm{~K}$ environment should be reached.
}

$1 / \mathrm{e}^{3}$. Taking into account the transmission of the injection device and the elimination of the ghost beams, about $150 \mathrm{~mW}$ are reaching the beam-splitter.

The visible source is a tunable dye laser (rhodamin $6 \mathrm{G})$, pumped by a continuous argon laser. This dye laser is used in the red part of the spectrum where its power is a few tens of milliwatts. The beam is brought to the experiment bench by a single mode optical fibre that allows an additional cleaning of the beam (the dye laser is in a separate room). At the fibre output, the beam is collimated by a microscope objective. The mixture IR/Visible is performed on a dichroic plate located in front of the beam-splitter.

\subsection{Beam separation and recombination}

The beam-splitter and the beam-combiner have been designed to avoid multiple wave interferences (Perot-Fabry resonator) and the superposition of multiple reflections on the transmitted wavefront. The classical plane-parallel beam-splitter has been replaced by a device made of 2 prismatic windows, one is placed upside down with respect to the other, and separated by a gap that allows multiple reflections to escape without changing the direction of the principal beam. The $27 \mathrm{~mm}$ diameter, $7.5 \mathrm{~mm}$ thick windows, separated by $60 \mathrm{~mm}$ have an angle of 5 degrees that allows us to control the first extra reflections on a $12 \mathrm{~mm}$ beam (Fig. 2). These ZnSe plates are not coated, and are used near the normal incidence (5 degrees). They have been polished to an accuracy of $\lambda / 20$ peak to valley $(\lambda=632.8 \mathrm{~nm})$. The global efficiency of such a device at $10.6 \mu \mathrm{m}$ is about $47 \%$ for the transmitted beam and $17 \%$ for the reflected one. At $10.6 \mu \mathrm{m}$, the absorption of $\mathrm{ZnSe}$ is low. The size of the beam avoids localized concentrations of energy and a thermal gradient to develop in the material. The remaining $36 \%$ of the source energy is spread over all the ghost beams (reflections on the plates' interfaces) that propagate out of the main beam directions. The flux differences introduced by the beam-splitter are compensated by the beam-combiner. The difficulty of this solution is the handling of extra reflections that can either be used as control signals for the servo-control of the experiment or be trapped to avoid interferences with the main beam. The coherence length of the lasers is much higher than the experiment dimensions.

After alignment, the only degrees of freedom of the interferometer are the two degrees of rotation of the beamcombiner that allow an angular superposition of the wavefronts and the two degrees of rotation through a tip tilt correction device that allows lateral superposition of the wavefronts. Both angular and lateral position control devices are powered by piezo electric stacks that allow very fine settings and/or servo control. All the other optical elements are screwed onto aluminium plates after the settings have been done. 


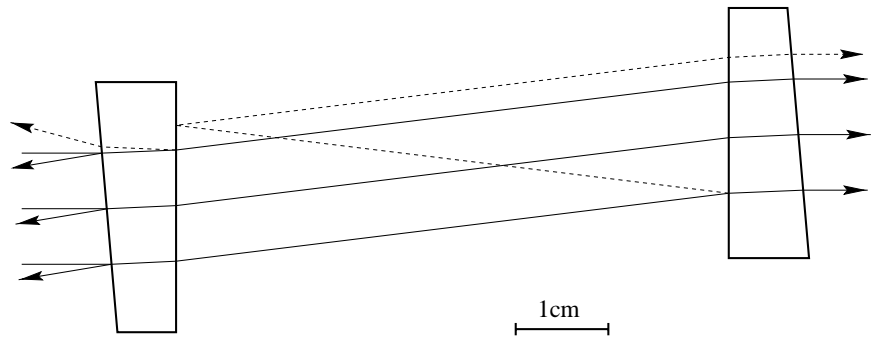

Fig. 2. Principle of the beam-splitter and the beam-combiner. We represent the main beam with plain lines and the first ghost reflections with dashed lines. $47 \%$ of the input energy is in the transmitted beam, $17 \%$ in the reflected one, and $36 \%$ is spread over all the ghost beams

\subsection{Wavefront quality control}

Various wavefront control and cleaning elements are added in the optical path:

- an infrared flux balance device;

- delay lines;

- a wavefront angular and lateral superposition device;

- an optical filtering device.

- The infrared flux balance device must equalize the intensity between the two arms of the interferometer before recombination.

This is a pure absorbing device, composed of two barium fluoride $\left(\mathrm{BaF}_{2}\right)$ prisms, one placed upside down with respect to the other. The first prism is fixed while the other is free to move beside it. The total thickness of absorbing material is thus constant over the whole beam section, and can be set within a range of $\pm 3 \%$ of the total transmission $\left(\tau_{i} \sim 0.72\right)$.

The flux balance device is set by successive measurements of each of the beam's intensity. The measurement of infrared intensity of each beam is performed by pyroelectric detectors with AR coated germanium windows (P5236 detectors from GEC-Marconi). These measurements are performed after recombination and optical filtering but before detection.

- The delay lines allow the localization and the control of the zero path difference with a precision of less than $10 \mathrm{~nm}$.

The optical part of a delay line is made by a cat eye, built with a parabolic primary mirror $(\phi: 50 \mathrm{~mm}$, focal length: $150 \mathrm{~mm}$ ) and a secondary plane mirror $(\phi 4 \mathrm{~mm})$. The secondary mirror is glued to a piezo-electric ceramic stack. It allows fast displacements over a total range of about 15 micrometers.

The cat eye is mounted on a frictionless table, which is necessary to control the displacements to a nanometer accuracy. As a consequence, this table involves only pure bending of metallic blades. Performances are:

\begin{tabular}{|l|l|}
\hline total course & $4 \mathrm{~cm}$ \\
\hline angular error & 3 arcsec (peak to valley) \\
\hline lateral displacement & 5 micrometers (peak to valley) \\
\hline
\end{tabular}

Linear motors provide low frequency translations (thermal drifts, slow fluctuations of path difference) while the piezo-electric ceramics provide high frequency (typically a few hundreds of $\mathrm{Hz}$ ) small translations (vibrations).

The optical path difference control is based on a polarization rotation sensitive system (Connes \& Michel 1975). A reference interferometer is included in the delay lines breadboard. It uses a helium-neon laser as a source and provides a signal proportional to the path difference with a resolution higher than $10 \mathrm{~nm}$ in a wide frequency band $(50 \mathrm{kHz})$.

The path difference servo control is made up of two loops: a "local" loop working with the He-Ne laser signal and a "vernier" loop working with the dye laser signal. The error signal is obtained through a lock-in detection of the visible dark interference pattern on the beam-combiner.

Finally, the servo controlled system (linear motor and piezo-electric ceramics) has a frequency band of $1.3 \mathrm{kHz}$, that reduces residual vibration effects at a level of $\sigma_{\mathrm{OPD}} \sim$ $5 \mathrm{~nm}$ (rms).

A PC type computer manages all the settings under LABVIEW and allows a flexible control of the system.

- The wavefront lateral superposition device is made up of a "tip-tilt" mirror located well ahead of the beam-combiner. It mainly changes the lateral position of one beam with respect to the other, allowing their superposition. This mirror however leads to a small angular deviation of one wavefront from the other. This deviation is compensated, as are the other sources, of deviation by a rotation of the beam-combiner itself, equipped with the same type of mount. The rotation of the beam-combiner modifies the orientation of only one of the two beams (the reflected one), and allows the angular settings of the system to be done. The other beam is taken as a reference. These devices are servo-controlled by error signals coming from beam lateral and angular position sensors (two CCD cameras monitoring the position at two different positions).

- Several optical filtering devices have been considered or are under evaluation.

- Pinholes: it is made up of a hole with a diameter comparable to the pupil Airy disk diameter (first intensity minimum of the diffraction pattern) obtained in focusing the beam from the interferometer after recombination. As mentioned before, the main drawback of this type of filter is that it is not efficient for low spatial frequency defects such as geometric distortions (the efficiency of a pinhole optical filtering increases with the defect's spatial frequency); 
- Another solution is to use single mode waveguides. Such waveguides are beginning to be available in that spectral range, particularly dielectric waveguides, and their huge absorption may still be acceptable given the small lengths and the powerful sources we use. The advantage of waveguides over pinholes is the possibility, at least in theory, to correct all phase defects (including low order defects such as tip-tilt, but not the optical path differences). Phase defects are transformed into coupling defects and path differences that can be monitored (Coudé du Foresto \& Ridgway 1991). Path difference fluctuations are corrected in both cases by delay lines. Coupling efficiency differences can be compensated for by the infrared flux balance device.

Single-mode waveguides for astronomy are already available (Kern \& Malbet 1996) in the visible and in the near infrared as single-mode fibres (Monerie et al. 1985) or integrated optics devices (Malbet et al. 1999). They should also soon be available in the thermal infrared range as fibres (Perrin et al. 2000), hollow waveguides, or integrated optics waveguides. These guides are manufactured by ion exchange in IR glasses (chalcogenides) and are under development (Laurent et al. 2000). One of the main difficulties is to reduce the loss in the guide, while still limiting its length to a few centimetres. However, considering a guide with a small index difference between core and cladding, it appears that a $1.5 \mathrm{~m}$ single-mode waveguide is necessary at $10 \mu \mathrm{m}$ to attenuate all the non-propagating modes by a factor of $10^{4}$ (Leproux et al. 2001).

To be efficient, the filtering must be symmetric on both optical paths of the interferometer. In the demonstration experiment we chose to put the filtering device after the beam-combiner. The two beams cross a unique and common device. Formally, the results are similar to separated identical devices because of the linearity of Maxwell's equations describing the recombination.

The filtering device is composed of focusing optics (this optical element is common to pinhole and waveguide devices), and relay optics to the detector.

\subsection{Detection}

The maximum power of the laser is $250 \mathrm{~mW}$. The total transmission of the interferometer is about $1.5 \%$. The intensity after recombination should thus vary from a few milliwatts (constructive interferences) to a few nanowatts (destructive interferences) with a rejection rate of about $10^{6}$. Such a dynamic range cannot be reached by common infrared detectors.

In our case, we want an accurate measurement of the destructive interference intensity. For low level detections, we choose to use $77 \mathrm{~K}$ HgCdTe detectors. These detectors were built by the SAT company and were used on the SOIRDETE interferometer (Mekarnia \& Gay 1990). They have a typical detectivity $D^{\star}=510^{10} \mathrm{~cm} \mathrm{~Hz}^{1 / 2} \mathrm{~W}^{-1}$ with a typical surface area of $50 \times 50 \mu \mathrm{m}^{2}$. These detectors were included in an Infrared
Lab cryostat by Jean Gay's team. The cryostat can function for about 24 hours without having to be refilled and this allows stable measurements to be made over a day. A custom made pre-amplifier allows accurate measurements.

\subsection{Mechanical stability of the experiment}

The experiment is implemented on a $4.2 \mathrm{~m} \times 1.0 \mathrm{~m}$ granite optical bench. The position of the 2.4 ton bench is servo-controlled with 3 fixed and 3 pneumatic devices. The beams are located $190 \mathrm{~mm}$ above the surface of the table. Every optical element is mounted on a barrel screwed onto a $100 \mathrm{~mm}$ long aluminium column (with a diameter of $36 \mathrm{~mm}$ ), which is screwed to an aluminium base and placed unscrewed on the marble bench on a kinematic device that allows smooth control of the position of the elements and avoids violent thermal relaxations. The temperature of the room varies over a range of 1 to 2 degrees during the course of a year. The stability of the whole device is good, although it has still not been quantified. However, fringes can be obtained over weeks without any supplementary modifications.

\subsection{Laboratory environment and turbulence}

The turbulence in the laboratory is harmful to the quality of measurements. It leads to local modifications of the air index on the optical paths and thus to permanent fluctuations of the global path difference (that could be corrected by delay lines) but also phase spatial fluctuations on the pupil. The path difference fluctuations should not be higher than $\lambda / 3000$ at $10 \mu \mathrm{m}$, to get a $10^{6}$ rejection rate. Turbulence appears as soon as a horizontal or a vertical (directed downwards) temperature gradient appears. In our case, a temperature difference between the table and the top cover of the experiment may exist.

This laboratory turbulence may be approximated, in the case of a vertical temperature gradient by a RayleighBénard problem (plane turbulence between two horizontal boundaries) and characterized by a number (the Rayleigh number) that is in fact the ratio of the potential energy to the viscous dissipation energy. This number, written $R a$, is given by the following relation:

$R a=\frac{g \cdot \alpha \cdot \Delta T \cdot h^{3}}{\kappa \cdot \nu}$

with:

$g$ : gravitational constant,

$\alpha$ : fluid thermal expansion coefficient,

$\Delta T$ : temperature difference,

$h$ : fluid layer height,

$\kappa$ : fluid heat diffusivity,

$\nu$ : fluid kinematic viscosity.

The appearance of convective cells exhibits a threshold for a particular value of the Rayleigh number, called the critical Rayleigh number and written $R a_{\mathrm{c}}$, of about 1700 in the case of a fluid layer between two horizontal solid 
boundaries (Drazin \& Reid 1981). This threshold appears when the diffusion is not fast enough to thermally balance the gas. Hot bubbles, that are lighter, appear and a general circulation starts in the experimental enclosure.

In the case of a horizontal temperature gradient, a circulation phenomenon also occurs, but this time, its onset does not exhibit any threshold. Particular care must be taken in order to avoid strong horizontal temperature gradients along the optical path (e.g. control of instrumental thermal dissipation).

In order to limit the effects of turbulence, the experiment is covered by a sealed helium, filled enclosure. This environment has two major advantages over air:

- The helium optical index fluctuations are 10 times lower than that in air when the temperature varies;

- The helium thermal conductivity is 6 times higher than that of air. This property allows a fast homogenization of temperature on all the optical paths, and delays the onset of turbulence (cf. Eq. (1)). As a comparison, the value of $R a_{\mathrm{c}}=1700$ is obtained with $h=30 \mathrm{~cm}$ for $\Delta T=6.910^{-4} \mathrm{~K}$ in air, while in helium $\Delta T$ must reach $4.510^{-2} \mathrm{~K}$ before turbulence appears.

The airtightness constraints are more or less the same as those used for a setup under low-quality vacuum conditions, with the huge difference that the enclosure structure does not need to support the effects of atmospheric pressure and so can be built with polymer (and transparent) materials; the pressure in the interior part of the enclosure is a bit higher $(\sim 2$ mbar $)$ than the pressure in the air.

Another solution to avoid the appearance of turbulent cells (which can be coupled to the former one) is to add an upward oriented temperature gradient in the enclosure. This gradient can be obtained by slowly heating $\left(\Delta T \sim 0.1-0.5^{\circ} \mathrm{C}\right)$ the top of the enclosure. Horizontal temperature gradient problems are not solved but this effect can be strongly minimized by reducing the internal heat sources as much as possible. In addition, the ascending thermal gradient tends to stop gas from moving when circulation starts.

Figure 3 gives a general view of the optical bench seen from the injection part towards the beam combiner device.

\section{First results}

\subsection{Visible interferences}

The entire system was designed to obtain high rejection rates (typically $10^{4}$ to $10^{6}$ ) at a wavelength of $10.6 \mu \mathrm{m}$. However, the first channel to be set and aligned was the visible channel (used later as a metrology channel for OPD and tip-tilt control of the infrared beam). We made several measurements on that channel.

A first measurement was performed with a "light" configuration (essentially beam-splitter and beam-combiner), without any other wavefront control device. With that configuration, it was not possible to obtain a stable flat

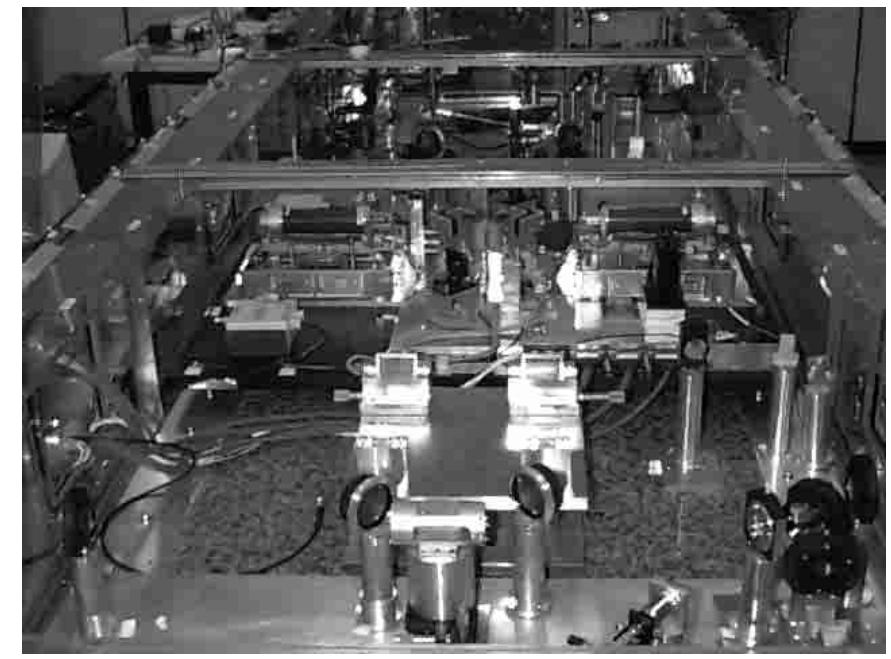

Fig. 3. General view of the testbed. From foreground to background: beam-splitter, flux balance device, delay lines, beamcombiner and optical filtering device

field. We could only measure the contrast of the fringe pattern. Such a measurement was performed with a photographic method described at the ICSO'97 and it exhibited a rejection rate of several hundreds (Ollivier et al. 1997).

\subsection{Infrared interferences}

The infrared channel and all the sub-systems were completed and implemented on the optical bench by the end of September 1999. The contrast measurements began just thereafter. However, further developments are still necessary to utilize the entire potential of the test-bed.

All the sub-systems were used but manually controlled. The contrast measurements were obtained from the following procedure:

- The interference state control is obtained manually by moving the delay lines with the control software. As the system is not servo-controlled by the visible channel, occasionally one must correct the optical path difference in order to keep the destructive interference state, in spite of the atmospheric effects which are present when the protective enclosure is open.

- The lateral and angular superpositions of the beams are set before the measurement, and they are not modified thereafter. These settings are obtained by the superposition of the two beams on two different points: the beam-combiner, and a point located far beyond it. To allow these settings, we use software that measures the photometric centre of mass of the beams, and then tip-tilt mirrors adjust the position. This system is a simplified version of the system described before.

- The flux balance device is set in neutral position. The theoretical transmission is the same in the two arms of the interferometer. Because the beams are not perfectly 

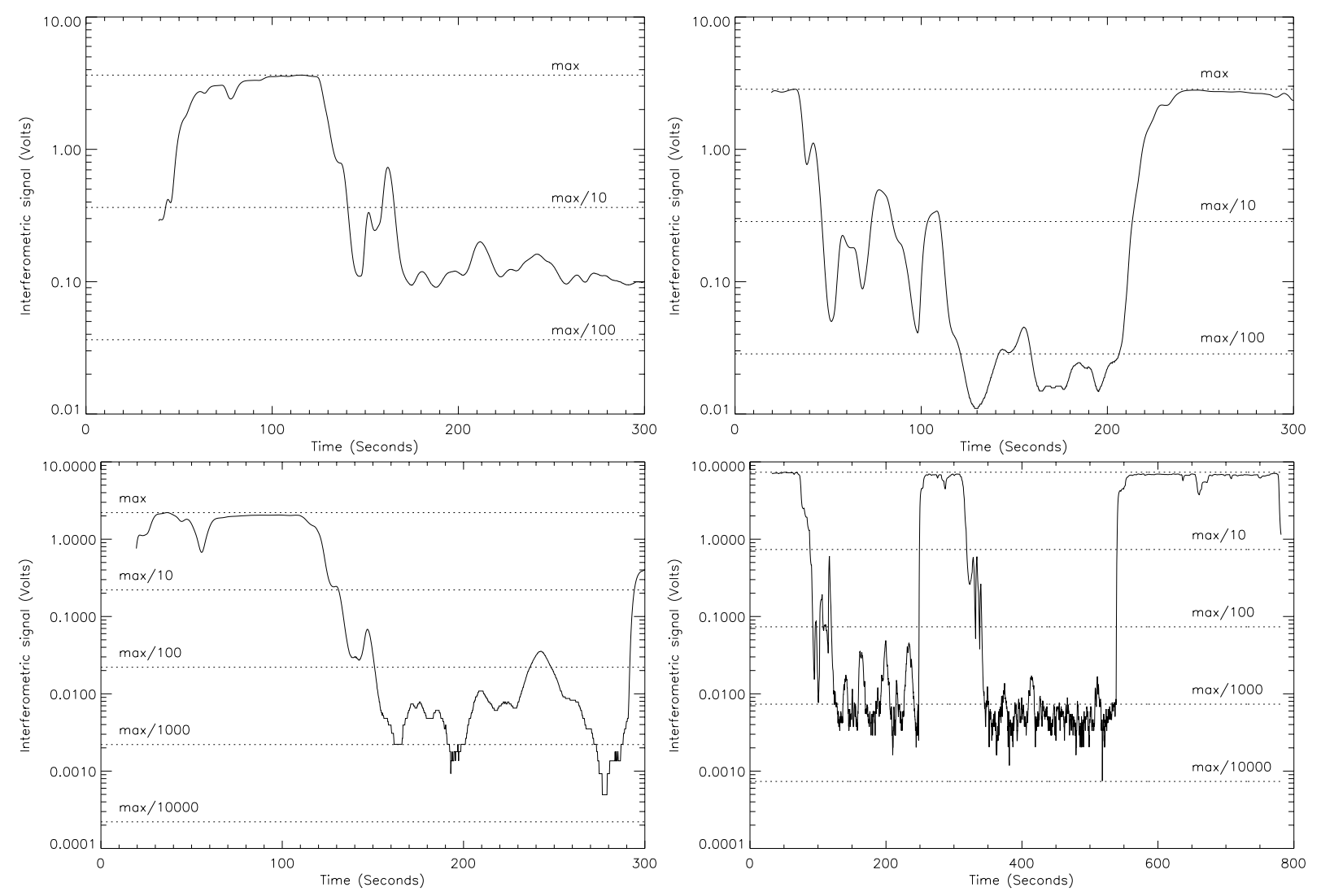

Fig. 4. Interferometric extinction as a function of time obtained with the interferometer by moving OPD from a bright to a dark fringe, without spatial filtering (top left) and with spatial filtering by a $200 \mu \mathrm{m}$ pinhole (top right), by a $50 \mu \mathrm{m}$ pinhole (bottom left) and by a $20 \mu \mathrm{m}$ pinhole (bottom right)

aligned while they propagate in the different arms, the photometric imbalance is still about $5 \%$ which is outside the range of the settings. This imbalance is due to the fact that the visible and infrared beams are not always laterally superposed, because the $\mathrm{ZnSe}$ beam-splitters have dispersion. Thus, it is difficult to control the exact position and diameter of the thermal infrared beam over the whole optical path. As the optical sub-systems are compact, their entrance window is hardly bigger than the size of the beam itself and vignetting can occur. This point will be improved upon later. Flux imbalance, visibility $(V)$ and rejection rate $(\rho)$ are linked by the following relation, assuming no phase mismatching:

$V=\frac{2 \sqrt{x}}{1+x}=\frac{\rho-1}{\rho+1}$.

Where $x=I 1 / I 2$, and $I 1$ and $I 2$ are the intensities in each arm of the interferometer. Assuming $x=0.95$, the biggest rejection rate that could be expected is about 6000. This flux imbalance does not preclude rejection rates of $10^{3}$, that are sufficient to demonstrate the relative improvement an optical filter provides. That's why we did not spend too much time improving that point for the first measurements. However, this question of alignment is crucial for improving the absolute value of the rejection rate.
- The detection chain is composed of:

- a chopper that modulates the signal just after the source at a frequency of about $1 \mathrm{kHz}$;

- a $100 \times 100 \mu \mathrm{m}^{2}$ single pixel HgCdTe detector cooled by liquid nitrogen, in a dewar that allows an angle of view of about 30 degrees;

- a pre-amplifier with a gain of about $10^{-7} \mathrm{~A} \mathrm{~V}^{-1}$;

- a lock-in amplifier with a 1 s time constant;

- an AD acquisition card, recording the 0-10 V coming from the lock-in amplifier (that is why all the graphs presented here have the same $Y$-axis unit). The quantification step of the $\mathrm{AD}$ converter can be seen at low level signals.

- The contrast measurements were performed as follows:

- a background signal was recorded with the source off;

- successive bright and dark interferences were recorded during several minutes;

- the contrast was calculated from these data.

The following four graphs (Fig. 4) show typical results obtained from the records. They show successive bright and dark interference levels, and the depth of the extinction. These graphs were obtained both with and without optical filtering by a pinhole, whose diameter was decreased, thereby increasing the contrast of the interference pattern as theoretically predicted (Ollivier \& Mariotti 1997). 


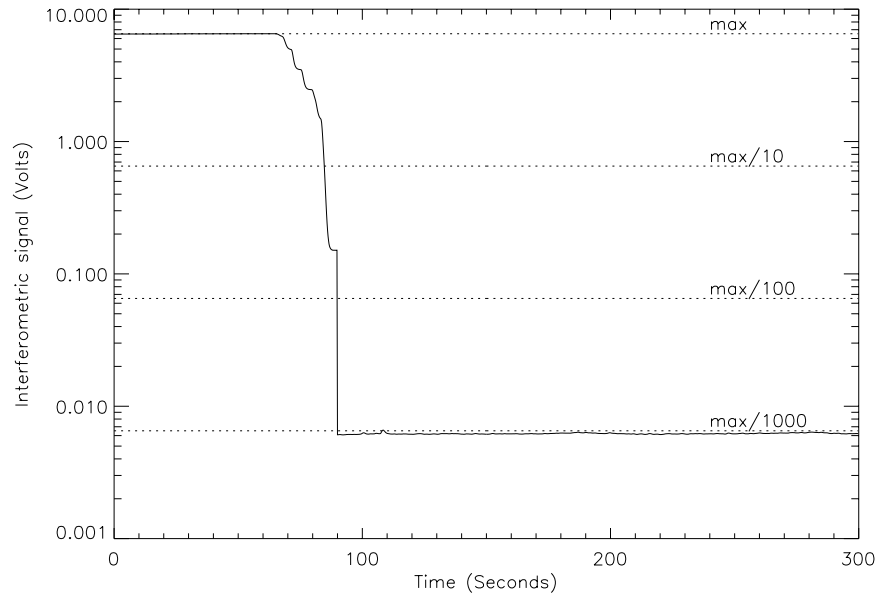

Fig. 5. Interferometric extinction obtained by changing the OPD with the protective enclosure closed and the top heated. The phase noise is strongly reduced and the rejection rate can be stabilized at a value of about 1000 without any active control of the OPD

With the same wavefronts, showing a flux inbalance of about $95 \%$, we increase the rejection rate from 40 (without filtering) to more than 1000 , which is at present the best value of extinction obtained by such a technique in the thermal infrared. This result clearly demonstrates the efficiency of the spatial filtering.

However, the maximum extinction seen as better than 1000 in Fig. 4 is obtained within large fluctuations. These fluctuations are typically due to phase variations over the optical paths due to turbulence. Note that the protective enclosure was open at the time of the measurements.

The next step is to stabilize the null. As mentioned before, there are two ways to do this and they can be done either separately or simultaneously:

- reduce the turbulence over the setup;

- actively control the OPD over all the optical paths.

At that level of extinction, only the first way was necessary to stabilize the interference pattern. The protective enclosure was closed, and its top was heated during 12 hours using heating patches. To reach values of rejection of about $10^{3}$, we found that a helium-filled enclosure was not necessary, since turbulence effects are negligible at that level of extinction. However, a helium filled enclosure remains necessary when high absolute values of rejection (typically $10^{5}-10^{6}$ ) are expected. The result is a cancellation of the turbulence over the setup as shown in Fig. 5 and the stability of the null. Note that the OPD is kept unchanged during the whole record.

A new series of developments and settings is under way to improve the rejection of this interferometer.

\section{Conclusion}

In this paper, we have presented a laboratory interferometer developed to obtain high contrast fringe patterns, and we demonstrate the validity of high extinction nulling interferometry in a monochromatic case.

This interferometer has been built. The first measurements exhibit extinction levels never before reached in this spectral range. This performance was obtained by the implementation of an optical filtering device whose principle had already been shown by numerical simulations, but had not previously been demonstrated in this wavelength range.

The next step is the development of existing setups, with the goal of demonstrating interferometric extinction for the polychromatic case. Then the development of an instrument that can be adapted to a ground-based interferometer, the Large Binocular Telescope (LBT) or the Very Large Telescope Interferometer (VLTI) for instance, can be considered.

Acknowledgements. The authors are very grateful to their colleagues, who took part in the definition and realization of the experiment. Special thanks to Patrick Bouchareine and Thierry Lépine from the Institut d'Optique Théorique et Appliquée in Orsay, Guy Artzner, Michel Decaudin, Patrick Boumier, Denis Barbet, Fernand Sarriau and Bruno Crâne from the Institut d'Astrophysique Spatiale in Orsay, Jean Gay and Yves Rabbia from the Observatoire de la Côte d'Azur, Vincent Coudé du Foresto, Guy Perrin, Bertrand Mennesson, Pascal Bordé and Patrick Dierich from the Département d'Études Spatiales in Meudon, Pierre-Olivier Lagage from the Service d'Astrophysique du CEA in Saclay, Fabien Malbet and Pascal Puget from the Laboratoire d'Astrophysique de l'Observatoire de Grenoble. The authors want to thank also Christian Chardonnet, Anne Amy-Klein and Alain Vanlerberghe from the Laboratoire de Physique des Lasers in Villetaneuse and Mr. Courtois from the Groupe de Spectrométrie Moléculaire de l'Université de Reims for their help in the field of $\mathrm{CO}_{2}$ lasers.

This work was supported by the Centre National d'Études Spatiales (CNES), the Centre National de la Recherche Scientifique (CNRS), the Université de Paris-Sud and the European Space Agency (ESA).

\section{References}

Angel, J. R. P., Cheng, A. Y., \& Woolf, N. J. 1986, Nature, 322,341

Bracewell, R. B. 1978, Nature, 274, 780

Connes, P., \& Michel, G. 1975, Appl. Opt., 14, 2067

Coudé du Foresto, V., \& Ridgway, S. 1991, FLUOR: a stellar interferometer using single-mode infrared fibres, in Proceedings of ESO Meeting on High-Resolution Imaging by Interferometry II, ed. J. M. Beckers, \& F. Merkle, European Southern Observatory, Garching bei München, 1991

Drazin, P. G., \& Reid, W. H. 1981, Hydrodynamic stability (Cambridge University Press)

Fridlund, M. 2000, DARWIN, The InfraRed Space Interferometer, The search for Terrestrial Exoplanets and High Resolution Imaging of the Universe, ed. M. Fridlund, ESA-SCI(2000)12

Hinz, P. M., Angel, J. R. P., Woolf, N. J., Hoffmann, W. F., \& McCarthy, Jr, D. W. 2000, SPIE Proc., 4006, 349 
Kern, P., \& Malbet, F. 1996, Integrated optics for astronomical interferometry, proceedings of Astrofib'96, ed. P. Kern, \& F. Malbet

Laurent, E., Schanen, I., Malbet, F., \& Taillades, G., Proc. SPIE, 4006, 1090

Léger, A. Mariotti, J.-M., Puget, J.-L., Rouan, D., \& Schneider, J. 1993, DARWIN mission concept

Léger, A., Mariotti, J.-M., Mennesson, B., et al. 1996, Could We Search for Primitiv Life on Extrasolar Planets in the Near Future? The DARWIN Project, Icarus, 123, 249

Leproux, P., Pagnoux, D., \& Reynaud, F., How to determine the fibre length for an accurate spatial filtering in a spatial interferometer, in preparation

Malbet, F., Kern, P., Schanen-Duport, I., et al. 1999, A\&AS, 138,135

Marcuvitz, N. 1951, Waveguide handbook (Dover Publications)

Mayor, M., \& Queloz, D. 1995, Nature, 37, 355

Mekarnia, D., \& Gay, J. 1990, A\&A, 238, 469

Mennesson, B., Ollivier, M., \& Ruilier, C. 2001, On the use of single-mode waveguides to correct the optical defects of a nulling interferometer, submitted to JOSA
Monerie, M., Alard, F., \& Maze, G. 1985, Electr. Lett., 21, 1179

Morgan, R. M., Burge, J. H., \& Woolf, N. J. 2000, SPIE Proc., 4006, 340

Ollivier, M., \& Mariotti, J.-M. 1997, Appl. Opt., 36, 5340

Ollivier, M., Mariotti, J.-M., Brunaud, J., et al. 1997, Coronographie Interférentielle pour la Mission Spatiale DARWIN - Expérience de Validation en Laboratoire, in proceedings of ICSO'97, Toulouse, France

Ollivier, M. 1999, Contribution à la recherche d'exo-planètes - Coronographie interférentielle pour la mission DARWIN, Ph.D. Thesis, Université de Paris-Sud, Paris XI

Perrin, G., Ollivier, M., \& Coudé du Foresto, V., Proc. SPIE, 4006, 1007

Schneider, J. 2000, The Extra-solar Planets Encyclopedia, http://www.obspm.fr/planets

Serabyn, E, 1999, Experimental confirmation of deep nulling, in Darwin and Astronomy, ESA-SP, 451, 101

Wolszczan, A., \& Frail, D. 1992, Nature, 255, 145

Woolf, N. J., \& Angel, J. R. P. 1998, ARA\&A, 36, 507 